\title{
A Method of Design Improvement with the Structured Product Concept
}

\author{
Ichiro Hirata $^{1}$ and Toshiki Yamaoka ${ }^{2}$ \\ ${ }^{1}$ Product Innovation Dept, Hyogo Prefectural Institute of Technology, \\ 3-1-12, Suma-Ku, Kobe City, Hyogo, Japan \\ ichiro@hyogo-kg.go.jp \\ ${ }^{2}$ Department of Design and Information Sciences, Faculty of Systems Engineering, \\ Wakayama University \\ 930 Sakaedani, Wakayama City, Wakayama, Japan \\ yamaokalsys.wakayama-u.ac.jp
}

\begin{abstract}
The product development in service science will become important for manufacturing industry. Therefore, the introduction of the "service science" concept is necessary in product development. This paper proposes a design improvement method based on HCD (Human Centered Design) concept, which can be introduced to middle/small enterprises, with the structured product concept. A case study of the operation panel design of home security system is discussed in this paper. This method is effective not only in making new products but also in improving developed products. Making a structured product concept is also effective to get consensus among the developers.
\end{abstract}

Keywords: Human Centered Design, Usability, Product concept.

\section{Introduction}

It is necessary to offer attractive products to get customer satisfaction. HCD (Human Center Design) is one of the techniques to gain user's satisfaction, and happiness on developing the products. In view of service engineering, it is important to adjust design and specification to yield the satisfaction. Therefore, HCD method can be used also for service science.

Since HCD has been recognized as a powerful tool for service science, large enterprises have been introducing HCD method and facilities. But, it is hard to introduce present HCD methods to middle/small enterprises because introducing HCD method is rather expensive. Thus, we have studied a design method to introduce HCD into middle/small enterprises.

In this paper, we suggest a method of design improvement with the structured product concept. This method makes it possible to introduce HCD into middle/small enterprises. The usability of products that were developed without the product concept also can be improved in terms of the product's usability by this method. 


\section{A Method of HDT (Human Design Technology)}

HDT is a logical product development and Human-centered Design method easily accessible to anyone. Defined as "Method to integrate Ergonomics, Industrial design, Marketing research, Cognitive science, Usability engineering, and Statistics (Multivariate analysis), to review process of product development to rely on intuition in the past by aspect of quantification as much as possible, and to support product making with charm of man priority that examines". HDT is developed in the following process.

\section{Step(1) Gather user requirements}

Extracting problems and needs related to a product. This usually involves group interviews, observation and task analysis.

\section{Step(2) Grasp current circumstances}

Investigating how users perceive a target product in the market.

\section{Step(3) Formulate structured concepts}

Constructing structured concepts based on user requirements and other types of information. Since the main specifications must be determined at this stage, structured concept should be structured for logical continuity among their various items, thereby avoiding any omissions. The weighting of the different concept items is particularly important as a measure to ensure logical continuity among them. This is also significant for revealing the items that are important. Once the items are weighted, those that should take precedence may be determined automatically when certain design items must be traded off against one another.

\section{Step(4) Design (synthesis)}

Visualizing a product based on the structured concepts. HDT requires that the design be based on the seventy predetermined design items. The seventy design items of

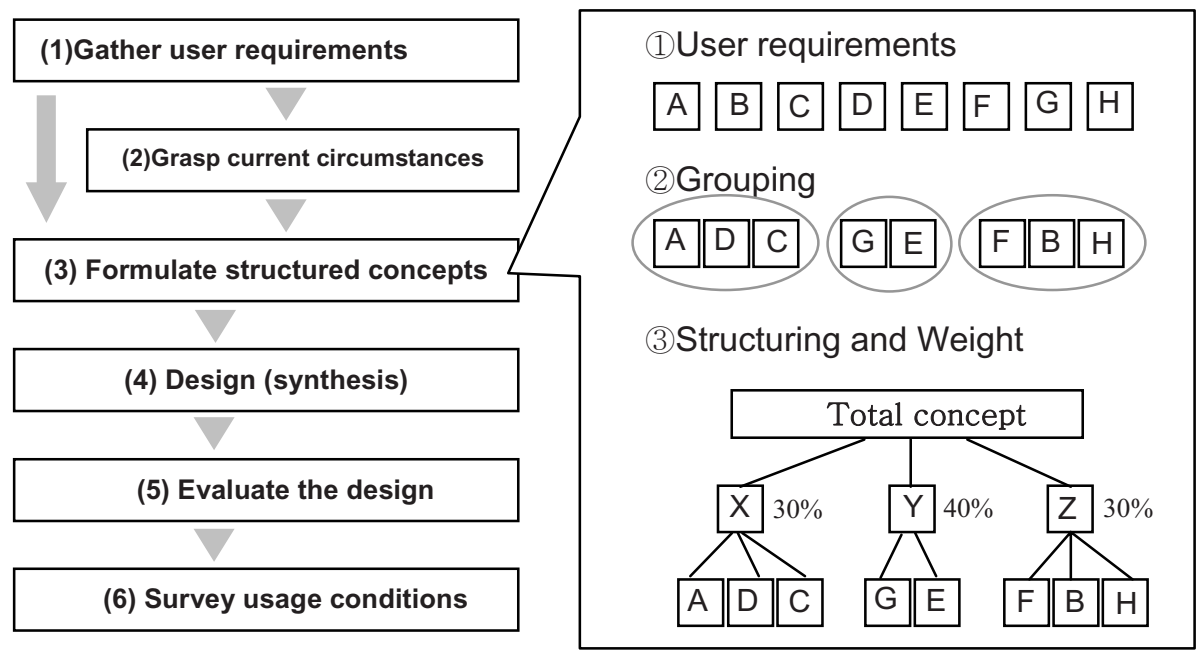

Fig. 1. Design process by HDT 
HDT are classified into the eight large groups: 1) User interface design (twenty-nine design items), 2) UD (nine design items), 3) Kansei (sensitivity) design (nine design items), 4) Product liability design (six design items), 5) Ecological design (five design items), 6) Robust design (five design items), 7) Maintenance design (two design items), and 8) other (human-machine interface design) (five design items).

\section{Step(5) Evaluate the design}

Evaluating the plan (design) as visualized.

\section{Step(6) Survey usage conditions}

Investigating purchaser attitudes towards the resulting product to identify needs for future product development.

\section{The Case Study of the Panel Design Improvement}

\subsection{Outline of the Home Security System}

The home security system illustrated in Fig 2. was developed by a middle/small enterprise in Hyogo prefecture, Japan. The concept was "an easy operation with a large operation panel". It is able to operate with the touch panel of 17 inches. It is set up in the entrance. "Floor plan of the room" besides the operation button and "Image of the web camera" and "Web page" are displayed on the screen. The state of lock can be confirmed with the floor plan of the room. When windows of the house open without permission, an emergency signal is send to the owner's cellular phone and images of the situation are recorded in the home security system's server. Moreover, it has the web browse function and the remote consumer electronic function besides the security function. In addition, it is possible to upgrade the software of contents in this product.

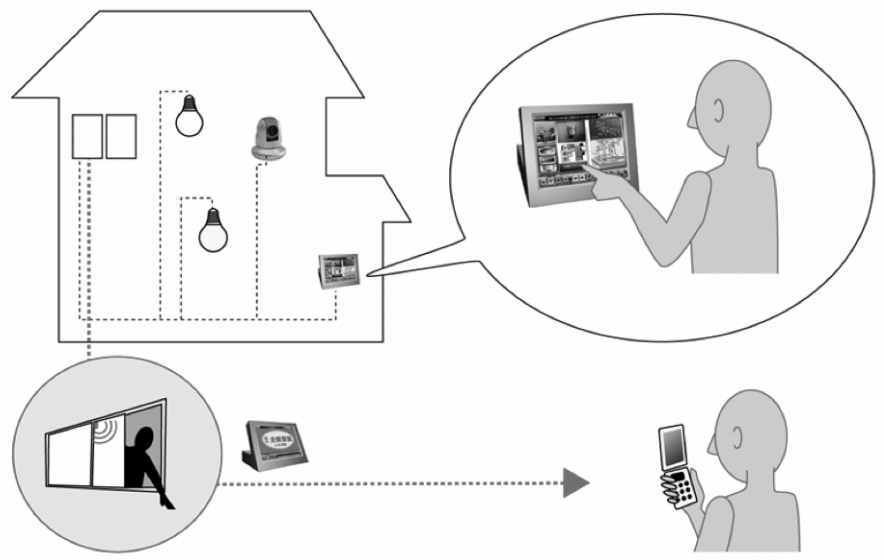

Fig. 2. A home security system 


\subsection{Improvement Based on HDT}

\section{Step1. Constructing structured product concept}

The designing process with the general product concept sometimes has the problem which item should be chosen if the items have the trade off relation. We can't determine the items if they don't have the priority. In addition, the detailed development policy is not shared among the developers with the general product concept.

A method of the structured concept was effective to solve these problems because it gives priority to some items for improvement. First, the characteristic items were examined from the product. These items were grouped by relation to make hierarchy. After that, weight is put to each item so that the total weight of the items becomes 100. The priority of each item was described as the weight in this process.

In this case, the target product has been made without the structured concept. We have aimed at the proposal of the technique for improving a present product. There was only a rough product concept when it was made. Therefore, the design items were resolved by observation on the feature of the product and then the structured concept was reconstructed.

6 items were extracted from the characteristic of the operation panel of the home security system (Fig 3.). These items were grouped in 3 items and distributed the weight to each item.

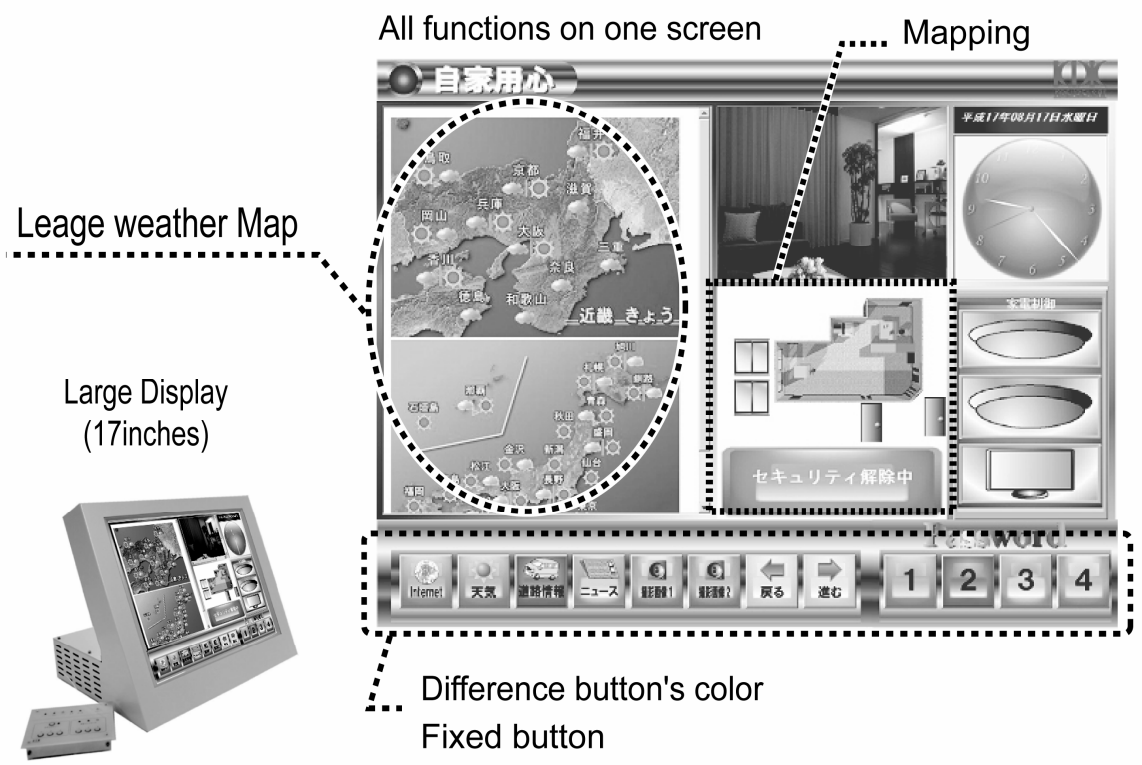

Fig. 3. Past panel design

\section{Step2. Evaluation of the product's usability and verification by task analysis}

To evaluate the operation panel's usability, task analysis was made to extract problems on the panel. On the task analysis, scene displayed in some situation was assumed. Problems of this scene were extracted from five view point of Human 


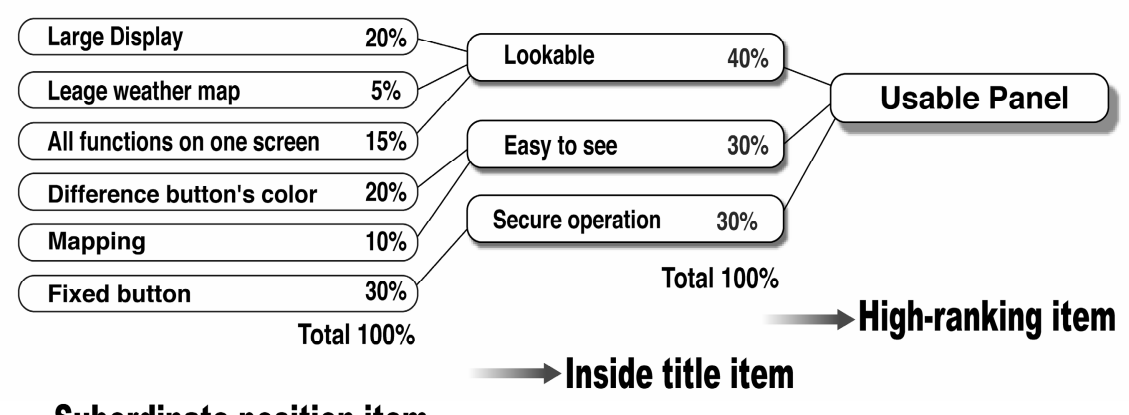

Subordinate position item

Fig. 4. Structured Product Concept (Past Design)

Machine Interface (Physical aspect, Information aspect, Temporal aspect, Environmental aspect, and Organizational aspect). The method of the extraction from three sides "Access to information", "Understanding and judgment", and "Operation" is acceptable according to the product.

79 problems about the product usability were found on the past design by task analysis.

\section{Step3. Validation by User test}

User test was conducted to verify the problems extracted by the task analysis. A protocol analysis, questionnaires and interview were made on 5 people.

The feature of this improvement process was to assume the product concept from the feature of the target product that has already been developed, and to verify the consistency of the design and concept. The feature of the present product became clear with this method, and the correction and the improvement points were found.

\section{Step4. Constructing the structured product concept of design improvement}

The problems extracted from the task analysis and the user tests were made into groups. The priority level was put on the problems, and the improvement plan of the item with a high priority level was made. After the made settlement plan was compared with the present product concept, the structured concept was corrected as an improvement concept (Fig 5.).

\section{Step5. Design and make prototype}

The design improvement idea (Fig 6.) was made based on the improvement product design concept. To conduct user test for evaluation of the design improvement idea, the prototype was made.

\section{Step6. Evaluating Design idea}

Evaluated the design improvement idea by user test. A protocol analysis, questionnaires were made on 5 people who were different from Step3 with a same protocol analysis task as Step 3.

Results of the evaluation, it found that past design's problems were solved by the design improvement idea. 


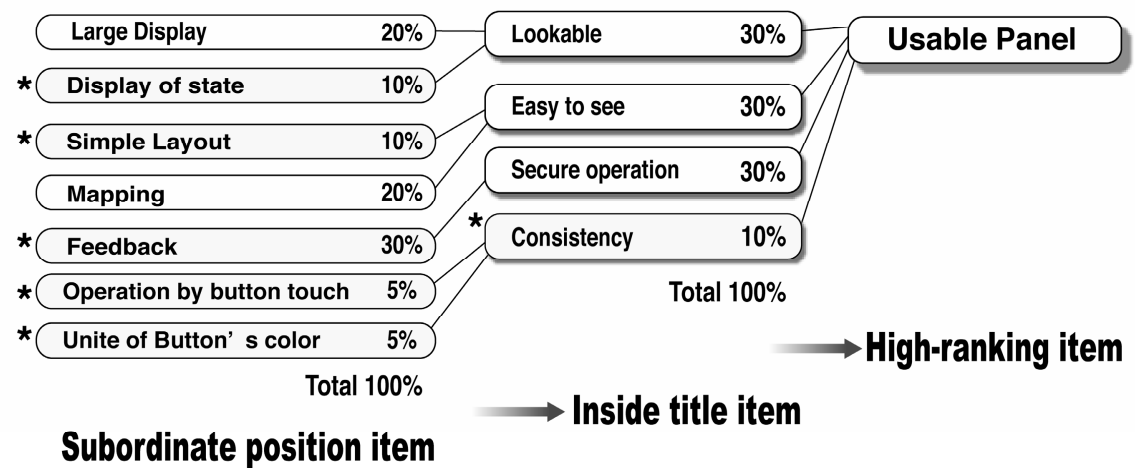

Fig. 5. Structured product concept of design improvement (* Item that is changed or added)

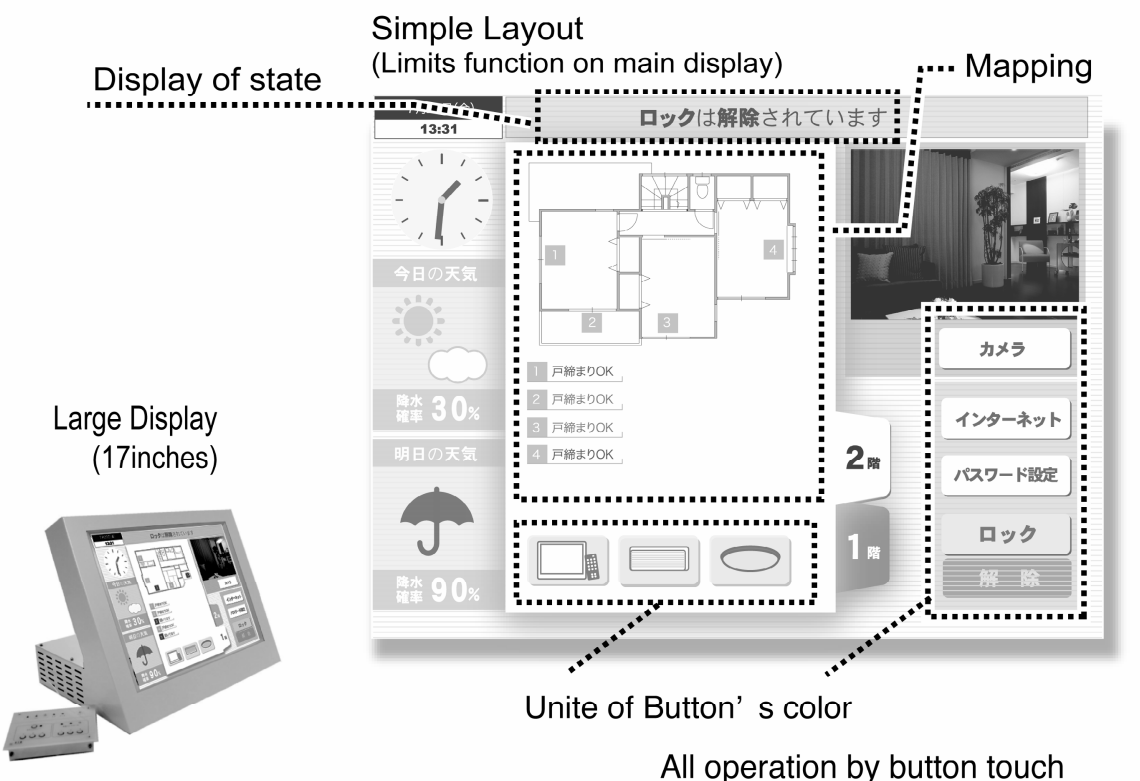

Fig. 6. The design improvement idea

\section{Conclusions}

To verify the effectiveness of the design improvement method with the structured product concept, we improved the operation panel of the home security system by this method. As a result, problems that have been extracted to evaluation of the past product could be almost solved. This method is effective for products that have been already developed in addition to make new products. Moreover, to make structured 
product concept is found to be effective for getting consensus among the developers in addition to the extraction of problems in the product.

In addition, the structured concept can give priority to items by putting different weights to them. This method can be applied to service science in addition to product development.

\section{References}

1. Kurosu, M.: Usability. Usability of Information Service. Science and technology of information (in Japanese) 54(8), 384-390 (2004)

2. Sakao, T., Shimomura, Y.: Service Engineering. A Novel Engineering Discipline for Producers to Increase Value Combining Service and Product, Journal of Cleaner Production, Special issue on sustainable production and consumption - making the connection (2006)

3. Sakao, T., Shimomura, Y.: Service Engineering. MVC (Model for Balancing Values and Costs). A Fundamental Model to Design Environmentally Conscious Services. In: Proceeding of the 4th International Symposium on Environmentally Conscious Design and Inverse Manufacturing (Eco Design 2005), IEEE Computer Society, Tokyo (2005)

4. Matsumoto, K., Tutaya, K.: Fujitsu's Activities for Achieving Universal Design. Fujitsu (in Japanese) 54(3), 168-172 (2003)

5. Yamaoka, T.: Introduction of Human Design Technology (in Japanese), vol. 4. Morikita Publisher, Tokyo (2003)

6. Yamaoka, T.: Introduction of Human Design Technology. Morikita Publisher, Tokyo(in Japanese), pp. 15-19 (2003)

7. Hirata, I., Okada, M., Kumada, T., Shimono, F., Yoshikawa, Y., Yamaoka, T.: Case of panel design improvement with Usability eveluation. The reseach forum for Gerontechnology, Japan (in Japanese), pp. 25-28 (2005) 